\title{
Anomalous versus Normal Room-Temperature Diffusion of Metal Adatoms on Graphene
}

\author{
Victor Gervilla,* Mohammad Zarshenas, Davide G. Sangiovanni, and Kostas Sarakinos
}

Cite This: J. Phys. Chem. Lett. 2020, 11, 8930-8936

Read Online

ABSTRACT: Fabrication of high-performance heterostructure devices requires fundamental understanding of the diffusion dynamics of metal species on 2D materials. Here, we investigate the room-temperature diffusion of $\mathrm{Ag}, \mathrm{Au}, \mathrm{Cu}, \mathrm{Pd}, \mathrm{Pt}$, and $\mathrm{Ru}$ adatoms on graphene using $a b$ initio and classical molecular dynamics simulations. We find that $\mathrm{Ag}, \mathrm{Au}, \mathrm{Cu}$, and Pd follow Lévy walks, in which adatoms move continuously within $\sim 1-4 \mathrm{~nm}^{2}$ domains during $\sim 0.04 \mathrm{~ns}$ timeframes, and they occasionally perform $\sim 2-4 \mathrm{~nm}$ flights across multiple surface adsorption sites. This anomalous diffusion pattern is associated with a flat $(<50 \mathrm{meV})$ potential energy landscape (PEL), which renders surface vibrations important for adatom migration. The latter is not the case for $\mathrm{Pt}$ and $\mathrm{Ru}$, which encounter a significantly rougher PEL $(>100 \mathrm{meV})$ and, hence, migrate via conventional random walks. Thus, adatom anomalous diffusion is a potentially important aspect for modeling growth of metal films and nanostructures on $2 \mathrm{D}$ materials.

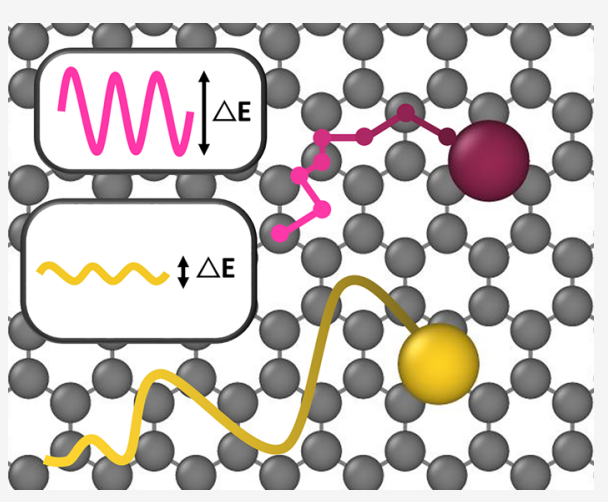

$\mathrm{F}$ abrication of multifunctional metal contacts in hetero- structure devices based on two-dimensional (2D) materials necessitates growth of vapor-deposited metal films in a controlled fashion on $2 \mathrm{D}$ crystal substrates. ${ }^{1,2}$ A key step toward achieving this control is to fundamentally understand the dynamics of adatom surface diffusion, the atomic-scale process that leads to island nucleation and thereby largely sets the lateral length scale of film morphology.

During epitaxial metal and semiconductor growth, at temperatures that are typical for thin-film deposition experiments, adatom diffusion is described as a thermally activated chain of uncorrelated jumps, so that adatoms execute a random walk on the substrate surface. ${ }^{5}$ Within this framework, the diffusivity of an adatom transitioning between surface sites separated by a mean square distance $l^{2}$ at rate $\nu$ is calculated as $D=\frac{1}{4} l^{2} \nu$. The temperature-dependent jump rate $\nu$ is typically approximated by the Arrhenius equation, in which the static (i.e., $0 \mathrm{~K}$ ) surface migration barrier is determined by $a b$ initio or classical computational methods, ${ }^{6-16}$ while the jump attempt frequency is estimated via (quasi-)harmonic transition-state theory approaches. ${ }^{17,18}$ Alternatively, $D$ can be computed using Einstein's equation $\left\langle x^{2}(t)\right\rangle=4 D t$, where $\left\langle x^{2}(t)\right\rangle$ is the adatom mean square displacement (MSD) after time $t$, with $\left\langle x^{2}(t)\right\rangle$ being measured either experimentally or estimated via classical molecular dynamics simulations. ${ }^{5,19}$

At temperatures significantly higher than those used for thinfilm synthesis experiments, the above-described notion of random walk can be inadequate ${ }^{20-25}$ because $\left\langle x^{2}(t)\right\rangle$ may not vary as $\sim t$. Such nonlinear $\left\langle x^{2}(t)\right\rangle$ versus $t$ dependence is indicative of anomalous diffusion and has been attributed to vibrational entropy modifying the potential energy landscape (PEL) of the substrate surface, thereby allowing for long and correlated adatom jumps. $^{26-31}$ As $2 \mathrm{D}$ materials are intrinsically inert $^{32,33}$ they often interact weakly with adatoms and their PEL is markedly flat, so that the onset of anomalous diffusion may take place at temperatures that are within experimentally relevant ranges (e.g., room temperature). Moreover, 2D materials, owing to their reduced dimensionality and the smaller density of vibrational modes they host in their lattice, are intuitively expected to dissipate adatom energies less efficiently as compared to bulk crystals, which may further enhance the tendency for anomalous surface diffusion.

In the present work, we explore the propensity of metal adatoms for anomalous diffusion on $2 \mathrm{D}$ crystals at room temperature. To this purpose, we study surface migration dynamics of silver $(\mathrm{Ag})$, gold $(\mathrm{Au})$, copper $(\mathrm{Cu})$, palladium $(\mathrm{Pd})$, platinum $(\mathrm{Pt})$, and ruthenium $(\mathrm{Ru})$ adatoms on singlelayer graphene $(\mathrm{SLG})^{34}$ via $a b$ initio and classical (semiempirical) molecular dynamics simulations. The choice of the six metals is motivated by the fact that (i) the reported estimates of their static surface migration barriers on graphene range from 0.004 to $0.188 \mathrm{eV}^{35}$ and hence represent a multitude of expected dynamic diffusive behaviors and (ii)

Received: August 3, 2020

Accepted: September 28, 2020

Published: September 28, 2020 


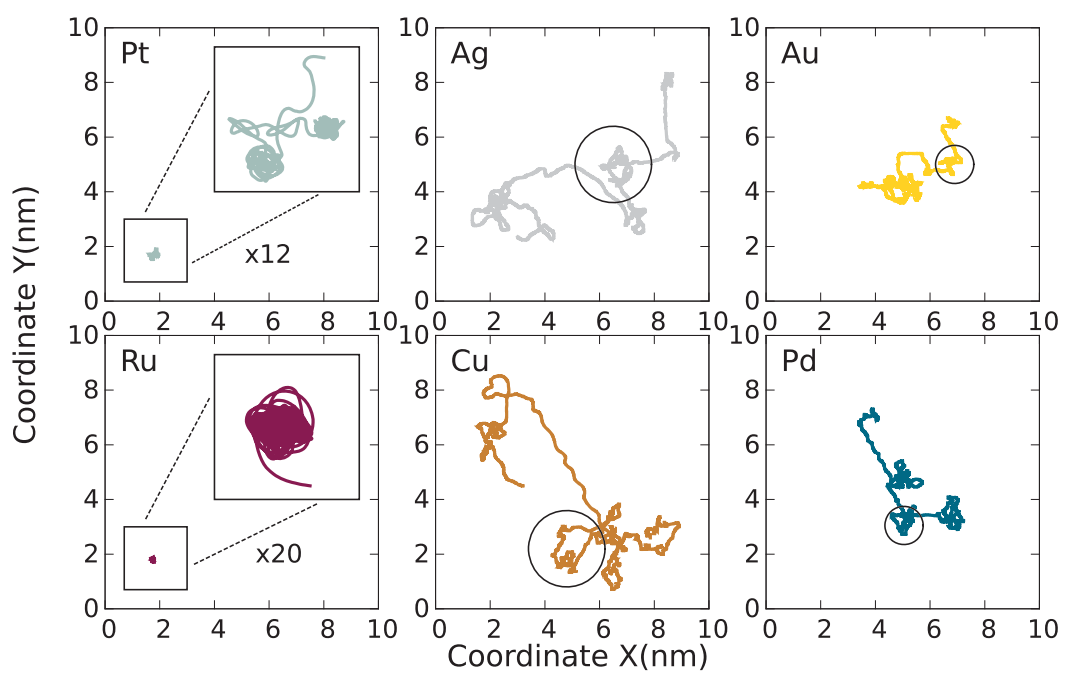

Figure 1. Ab initio MD diffusion trajectories for $\mathrm{Pt}(0.04 \mathrm{~ns})$; $\mathrm{Ru}(0.02 \mathrm{~ns})$; and $\mathrm{Ag}, \mathrm{Au}, \mathrm{Cu}$, and $\mathrm{Pd}(0.2 \mathrm{~ns})$. The circles in the trajectories for $\mathrm{Ag}$, $\mathrm{Au}, \mathrm{Cu}$, and Pd mark a short-range diffusion domain (see text for more details).

they are relevant as metal contacts in nanoelectronics, sensing, and catalysis. ${ }^{36-39}$

We first perform $a b$ initio molecular dynamics (AIMD) simulations of monomer diffusion on SLG for times up to 0.2 ns (see later in the text for simulation details and detailed explanation of the reasons behind the selection of the simulation times for the various adatoms). The trajectories are displayed in Figure 1, which shows that $\mathrm{Pt}$ and $\mathrm{Ru}$ diffuse within areas of $\sim 0.04-0.09 \mathrm{~nm}^{2}$. Closer inspection of the trajectories (enlarged sections presented as insets in the $\mathrm{Pt}$ and $\mathrm{Ru}$ trajectory panels) reveals that the adatoms spend most of the simulation trapped in specific surface sites, and for the case of $\mathrm{Pt}$, random jumps among neighboring adsorption sites are observed. All the above-described features are consistent with a random walk. In contrast, $\mathrm{Ag}, \mathrm{Au}, \mathrm{Cu}$, and $\mathrm{Pd}$ atoms move continuously across the surface without being halted by adsorption sites, diffuse within relatively short-range domains (marked with circles in Figure 1) during time frames of $\sim 0.04$ ns, and occasionally perform long jumps that cover multiple adsorption sites (the distance between adjacent adsorption sites is $\sim 0.12-0.24 \mathrm{~nm}$ ). This anomalous behavior corresponds to superdiffusion. Moreover, visual inspection of the trajectories reveals that both the areas of the domains and the jump lengths for $\mathrm{Ag}$ and $\mathrm{Cu}$ trajectories (the approximate mean values for these quantities are $\sim 4 \mathrm{~nm}^{2}$ and $\sim 4 \mathrm{~nm}$, respectively) are significantly larger than those for $\mathrm{Au}$ and $\mathrm{Pd}$ $\left(\sim 1 \mathrm{~nm}^{2}\right.$ and $\left.\sim 2 \mathrm{~nm}\right)$. The distinctly different normal versus superdiffusive behavior exhibited by the various adatoms is further illustrated in Figure S1 in the Supporting Information which plots trajectories of all metals after a $0.02 \mathrm{~ns}$ simulation time.

To estimate the diffusivity $D$ and quantify the migration dynamics of single adatoms, we compute, for each one of the anomalous trajectories in Figure 1, the time-averaged adatom mean square displacement ${ }^{40} \overline{\delta^{2}(\tau)}$

$$
\overline{\delta^{2}(\tau)}=\frac{1}{N} \sum_{n=0}^{N-1}|\boldsymbol{x}(n \Delta t+\tau)-\boldsymbol{x}(n \Delta t)|^{2}
$$

In eq $1, \Delta t$ is the simulation time step, $\tau$ a variable representing a specific observation time scale, and $N$ the total number of subtrajectories with a duration $\tau$ that can be defined over the total atomic trajectory (note that two different subtrajectories defined in this way may partially overlap). For a total simulation time $T$ and an observation scale $\tau$ consisting of $N_{T}$ and $N_{\tau}$ time steps $\Delta t$, respectively, $N=N_{T}-N_{\tau}+1$. Thus, each point on a $\overline{\delta^{2}(\tau)}$ versus $\tau$ curve corresponds to an average entailing information on all subtrajectories with a duration $\tau$. From the latter it follows that the statistical significance of a given $\overline{\delta^{2}(\tau)}$ value decreases with increasing $\tau$, because larger $\tau$ values yield a smaller number of subtrajectories available for calculating $\overline{\delta^{2}(\tau)}$. Moreover, it is worth pointing out that the analysis on the data in Figure 1 is based on $\overline{\delta^{2}(\tau)}$, instead of $\left\langle x^{2}(t)\right\rangle$, because an accurate calculation of the latter quantity would require data from multiple simulated trajectories.

The $\overline{\delta^{2}(\tau)}$ versus $\tau$ curves $(0<\tau \leq 50 \mathrm{ps}$; a detailed explanation for the selection of $\tau$ range is provided later in the text) for $\mathrm{Ag}, \mathrm{Au}, \mathrm{Cu}$, and $\mathrm{Pd}$ are plotted in log-log scale in Figure 2. The data reveal that $\mathrm{Ag}$ and $\mathrm{Cu}$ exhibit larger $\overline{\delta^{2}(\tau)}$ values over the entire $\tau$ range, which is in line with the differences in the jump length magnitudes seen in Figure 1. Furthermore, irrespective of the atomic displacement magnitude, the $\overline{\delta^{2}(\tau)}$ versus $\tau$ curves exhibit a nonlinear behavior

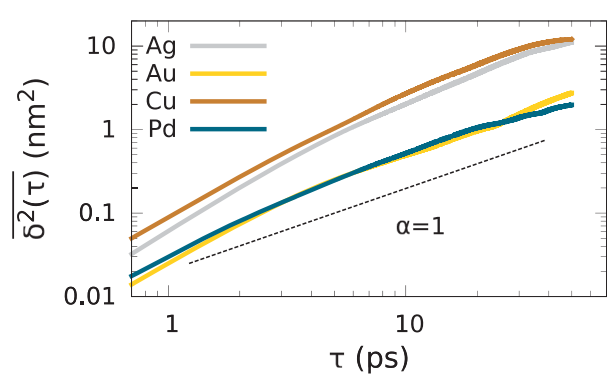

Figure 2. $\overline{\delta^{2}(\tau)}$ versus $\tau$ curves (plotted in log-log scale) extracted from the AIMD trajectories presented in Figure 1 for $\mathrm{Ag}, \mathrm{Au}, \mathrm{Cu}$, and Pd. All lines exhibit a slope $\alpha>1$ which is consistent with the superdiffusive nature of adatom motion. This is in contrast to random walk trajectories which exhibit a $\overline{\delta^{2}(\tau)}$ versus $\tau$ slope of $\alpha=1$ (represented by the dashed line). A more detailed discussion on the correlation among $\alpha$ and diffusion nature can be found in the text. 
Table 1. Exponents $\alpha$, Diffusivities $\left(D_{\alpha}, \mathrm{nm}^{2} \cdot \mathrm{ps}^{-\alpha}\right)$, and Adsorption Energies at 0 and $300 \mathrm{~K}\left(E_{\text {ads }}^{0 \mathrm{~K}}\right.$ and $E_{\mathrm{ads}}^{300 \mathrm{~K}}$, meV) of Metal Adatoms on the Graphene Surface ${ }^{a}$

\begin{tabular}{|c|c|c|c|c|c|c|c|c|}
\hline & \multirow[b]{2}{*}{$\alpha$} & \multirow[b]{2}{*}{$D_{\alpha}$} & \multicolumn{3}{|c|}{$E_{\mathrm{ads}}^{0 \mathrm{~K}}$} & \multicolumn{3}{|c|}{$E_{\mathrm{ads}}^{300 \mathrm{~K}}$} \\
\hline & & & hollow & bridge & top & hollow & bridge & top \\
\hline $\mathrm{Ag}$ & $1.4\left(\times 1.07^{ \pm 1}\right)$ & 0.016 & 9 & 1 & 0 & 6 & 0 & 3 \\
\hline $\mathrm{Au}$ & $1.2\left(\times 1.08^{ \pm 1}\right)$ & 0.007 & 22 & 5 & 0 & $\sim 33$ & 6 & 0 \\
\hline $\mathrm{Cu}$ & $1.4\left(\times 1.07^{ \pm 1}\right)$ & 0.024 & 184 & 0 & 8 & $\sim 171$ & 4 & 0 \\
\hline $\mathrm{Pd}$ & $1.1\left(\times 1.09^{ \pm 1}\right)$ & 0.008 & 290 & 0 & 53 & $\sim 660$ & 0 & 19 \\
\hline $\mathrm{Pt}$ & 1 & & 894 & 0 & 215 & & & \\
\hline $\mathrm{Ru}$ & 1 & & 0 & 641 & 664 & & & \\
\hline
\end{tabular}

${ }^{a}$ The adsorption energies refer to the hollow, bridge, and top positions, whereby energies are shifted with respect to the most stable adsorption site for which $E_{\text {ads }}$ is set equal zero.

with slopes $\alpha$ larger than one (the $\alpha=1$ slope is represented by the dashed line in Figure 2), which is consistent with the superdiffusive nature of the atomic trajectories in Figure $1 .^{41}$ Note that $\alpha \approx 1$ as $\tau \rightarrow 50$ ps. We attribute this to the limited statistical significance of $\overline{\delta^{2}(\tau)}$ at large $\tau$ values, e.g., $\overline{\delta^{2}(\tau)}$ for $\tau$ $=50 \mathrm{ps}$ is calculated using 4 subtrajectories (see eq 1 ).

On the basis of the nonlinear behavior established in Figure 2, we conclude that we cannot use the Einstein equation for correlating $\overline{\delta^{2}(\tau)}$ versus $\tau$ curves and $D$. For this reason, in the remainder of the Letter we use the continuous time random walk formalism as an alternative framework to treat anomalous diffusion. $^{42}$ This formalism assumes that $\overline{\delta^{2}(\tau)}$ exhibits a power law dependence on time

$$
\overline{\delta^{2}(\tau)} \simeq 4 D_{\alpha} \tau^{\alpha}
$$

where the exponent $1<\alpha<2$ denotes the superdiffusive character of the monomer ( $\alpha=2$ corresponds to a ballistic trajectory, whereas $\alpha=1$ corresponds to a random walk) and $D_{\alpha}$ is a generalized diffusion coefficient with physical dimensions $\mathrm{nm}^{2} \cdot \mathrm{ps}^{-\alpha}$.

The task of extracting relevant values for $\alpha$ and $D_{\alpha}$ is not straightforward, because each point at the $\overline{\delta^{2}(\tau)}$ versus $\tau$ curves has a different statistical significance (see eq 1 ). Thus, following the methodology proposed by Saxton, ${ }^{40}$ only $\overline{\delta^{2}(\tau)}$ points within an interval [0: $\left.\tau_{\text {cut }}\right]$ are considered. The value $\tau_{\text {cut }}$ is a time cutoff here taken to be equal to 50 ps (see Figure 2), to exclude all points averaged over less than 4 independent subtrajectories. Moreover, this choice has a physical justification: $\tau_{\text {cut }}$ is of the same order of magnitude as the typical time required for an adatom to transition between superdiffusive domains-note that the trajectories in Figure 1 consist of $\sim 3-4$ domains - and is thus representative of the adatom migration dynamics at the time scale of several tens of picoseconds. We also notice in Figure 2 that the slope of log$\log \overline{\delta^{2}(\tau)}$ versus $\tau$ curves is not constant. Hence, in order to extract $\alpha$ we perform a linear fit of the curves for multiple time intervals [0: $\left.\tau^{*}\right]$ with $0<\tau^{*} \leq 50$ ps as explained by Kepten et al. ${ }^{43}$ Then, the most probable value from the statistical distribution of $\alpha$ is selected (see Figure S2 in the Supporting Information), from which $D_{\alpha}$ is calculated using eq 2 .

Table 1 lists the numerical values of $\alpha$ and $D_{\alpha}$ for $\mathrm{Ag}, \mathrm{Au}$, $\mathrm{Cu}$, and $\mathrm{Pd}$. With regards to the scaling exponents, we see that $\mathrm{Ag}$ and $\mathrm{Cu}$ have an exponent $\alpha=1.4$, whereas for $\mathrm{Au} \alpha=1.2$, and for $\operatorname{Pd} \alpha=1.1$. Table 1 also presents the static $(0 \mathrm{~K})$ adsorption energies $E_{\mathrm{ads}}^{0 \mathrm{~K}}$ (see later in the text for details) of all metals (Pt, $\mathrm{Ru}, \mathrm{Ag}, \mathrm{Cu}, \mathrm{Au}$, and $\mathrm{Pd}$ ) on the hollow, bridge, and top site of graphene (hollow, above center of a C6 ring; bridge, above midpoint of $\mathrm{C}=\mathrm{C}$ bond; top, above a $\mathrm{C}$ atom). The $E_{\mathrm{ads}}^{0 \mathrm{~K}}$ values are validated against high accuracy static relaxations and are compared to data for both SLG and multilayer graphene from the literature (see Table S1 in the Supporting Information). ${ }^{7,38,44,45} \mathrm{We}$ note that the energy values in Table 1 are shifted with respect to the most stable site for which $E_{\text {ads }}^{0 \mathrm{~K}}$ is set equal zero. By comparing $\alpha$ and $E_{\text {ads }}^{0 \mathrm{~K}}$, we conclude that the propensity of an adatom toward anomalous diffusion can be correlated with the flatness of the surface PEL: (i) for $\mathrm{Pt}$ and $\mathrm{Ru}$, the smallest energy difference between adsorption sites $\Delta E_{\text {ads }}^{0 \mathrm{~K}}$ is in the range $\sim 200$ to $\sim 600 \mathrm{meV}$, yielding a random walk behavior; (ii) while for $\mathrm{Ag}, \mathrm{Cu}, \mathrm{Au}$, and $\mathrm{Pd}, \Delta E_{\text {ads }}^{0 \mathrm{~K}}$ is considerably lower $(\sim 1$ to $\sim 50 \mathrm{meV})$, which offers more and easier transition pathways and promotes superdiffusive behavior, as seen by the $\alpha>1$ values. Moreover, the PEL can also be correlated with the degree of anomaly of the different metals that exhibit superdiffusion: the markedly flat $\mathrm{PEL}$ for $\mathrm{Ag}$ and $\mathrm{Cu}$ ( $\Delta E_{\mathrm{ads}}^{\mathrm{OK}}$ of the order of a few millielectronvolts) induces long atomic jumps, large diffusion domains ( $\sim 4 \mathrm{~nm}$ and $\sim 4 \mathrm{~nm}^{2}$, respectively), and $\alpha=1.4$; whereas the somewhat rougher PEL of $\mathrm{Au}$ and $\mathrm{Pd}\left(\Delta E_{\mathrm{ads}}^{\mathrm{OK}}\right.$ up to 1 order of magnitude larger) results in relatively smaller jump lengths and domain areas for $\mathrm{Au}$ and $\mathrm{Pd}\left(\sim 2 \mathrm{~nm}\right.$ and $\left.\sim 1 \mathrm{~nm}^{2}\right)$, and yields $\alpha=1.2$ and $\alpha=1.1$, respectively. Hence, by computing the static PEL of an adatom on a $2 \mathrm{D}$ material, we can qualitatively assess the adatom tendency for exhibiting anomalous diffusion patterns. However, it is important to emphasize that the adatom jump lengths and the specific dynamic diffusion behavior established for the various adatomon-SLG systems should be considered in relation to the mean separation between islands observed in experiments ${ }^{35}$

The normal (i.e., random walk) diffusive behavior of Pt and $\mathrm{Ru}$ adatoms at $300 \mathrm{~K}$ can be explained by the fact that the thermal energy $k_{\mathrm{B}} T(\approx 25 \mathrm{meV})$ is signifficantly smaller than the minimum $\Delta E_{\text {ads }}^{0 \mathrm{~K}}$ that separates stable adsorption sites. In contrast, the collective motion of substrate surface atoms may play a decisive role on the dynamics of adatoms interacting weakly with the substrate, whereby the minimum $\Delta E_{\mathrm{ads}}^{0 \mathrm{~K}}$ may become comparable with the thermal energy $k_{\mathrm{B}} T$. The latter is particularly relevant for strongly anharmonic materials like graphene, ${ }^{46}$ in which out-of-plane vibrations are relatively larger compared to those on surfaces of bulk crystals. To qualitatively understand the effects induced by graphene vibrations on the PEL, we calculate the adsorption energy of hollow, bridge, and top graphene sites at $300 \mathrm{~K}$ (see later in the text for details). Table 1 shows that, for the cases of $\mathrm{Ag}, \mathrm{Au}$, and $\mathrm{Cu}$, the numerical accuracy of DFT for adsorption-energy values (a few millielectronvolts) is comparable with the 
differences between $\Delta E_{\mathrm{ads}}^{0 \mathrm{~K}}$ and $\Delta E_{\mathrm{ads}}^{300 \mathrm{~K}}$. The influence of temperature is more pronounced for the case of $\mathrm{Pd}$, where $\Delta E_{\text {ads }}^{300 \mathrm{~K}}$ between bridge and top sites decreases by $\sim 30 \mathrm{meV}$ compared to $\Delta E_{\text {ads }}^{300 \mathrm{~K}}$. This temperature-induced effect may explain the fact that $\mathrm{Pd}$ exhibits a diffusion behavior fairly similar to that of $\mathrm{Au}$, despite having a notably larger $0 \mathrm{~K} \mathrm{PEL}$ corrugation $(\sim 50$ vs $\sim 5 \mathrm{meV})$. It is important to note that atomic vibrations do not only affect adatom migration dynamics by modifying the effective PEL; they may also alter the adatom preferences for occupying the different adsorption sites because of entropy effects, as explained in the Supporting Information (Section S4 and Table S2).

Besides AIMD, we employ classical molecular dynamics (CMD) to model multiple $\mathrm{Ag}$ trajectories of $3 \mathrm{~ns}$ and a long one of $30 \mathrm{~ns}$ (see later in the text for details). By using the same methodology as that in the AIMD simulations to analyze the 30 ns long trajectory (see Figure S3 in the Supporting Information), we extract a $\overline{\delta^{2}(\tau)}$ versus $\tau$ exponent $\alpha=1.3$, which shows that CMD also predicts anomalous diffusion behavior for Ag. This can be understood in light of the shallow PEL that CMD yields (see Table S3 in the Supporting Information), which is consistent with AIMD (i.e., millielectronvolt difference between adsorption sites). Because both simulation approaches yield a qualitatively consistent physical behavior, we take advantage of the higher computational efficiency of CMD, to gather statistics and further our understanding of the type of diffusion that adatoms perform on SLG.

We analyze the trajectories by computing the ensemble mean $\left\langle\overline{\delta^{2}(\tau)}\right\rangle$ of the time-averaged 3 ns trajectories and compare it with the $\left\langle\boldsymbol{x}^{2}(t)\right\rangle$ of the same trajectories in order to confirm or refute the ergodic character ${ }^{47}$ of the diffusion dynamics. Figure $3 \mathrm{a}$ shows that the two averages differ by a small factor from the simulations start until $0.1 \mathrm{~ns}$, after which they converge. This discrepancy at short and medium time scales indicates that, because of the tendency of adatoms to perform long jumps, the expansion rate of the area that an adatom may visit is greater than its mean diffusion rate. The adatom is, thus, unable to access all points of the phase space, which renders the motion nonergodic.

Moreover, we calculate the adatom jump length probability distribution

$$
p(l) \sim|l|^{-\mu}
$$

of the $30 \mathrm{~ns}$ run for time sampling intervals $\Delta t_{\mathrm{S}}=7.5,10,12.5$, and $15 \mathrm{ps}$, and fit a regression line for each of the $p(l)$ data sets, which are plotted in Figure $3 \mathrm{~b}$. For all the cases, the slope of the regression lines takes values $1.4<\mu<1.7$. We conclude from these results that the adatom motion is an intermediate case between ballistic motion $(\mu=1)$ and random walk $(\mu=$ 3).

The nonergodic character and the specific jump length distribution established by the analysis presented in Figure 3 indicate that $\mathrm{Ag}$ adatoms follow a fractal movement pattern known as Lévy walk, ${ }^{41,42,48}$ which is also observed in other phenomena in physical and biological sciences, including light propagation in optical media, ${ }^{49}$ animal foraging, ${ }^{50}$ or human travel. ${ }^{51} \mathrm{We}$ expect that $\mathrm{Au}, \mathrm{Cu}$, and Pd also perform a Lévy walk, owing to the qualitative resemblance of their AIMD trajectories to that of $\mathrm{Ag}$, as well as because for $\mathrm{Ag}, \mathrm{Au}, \mathrm{Cu}$, and $\mathrm{Pd}$ we find that $\alpha>1$. Lévy walks have an effective upper limit for their jump lengths, ${ }^{52}$ which allows treating the correlated
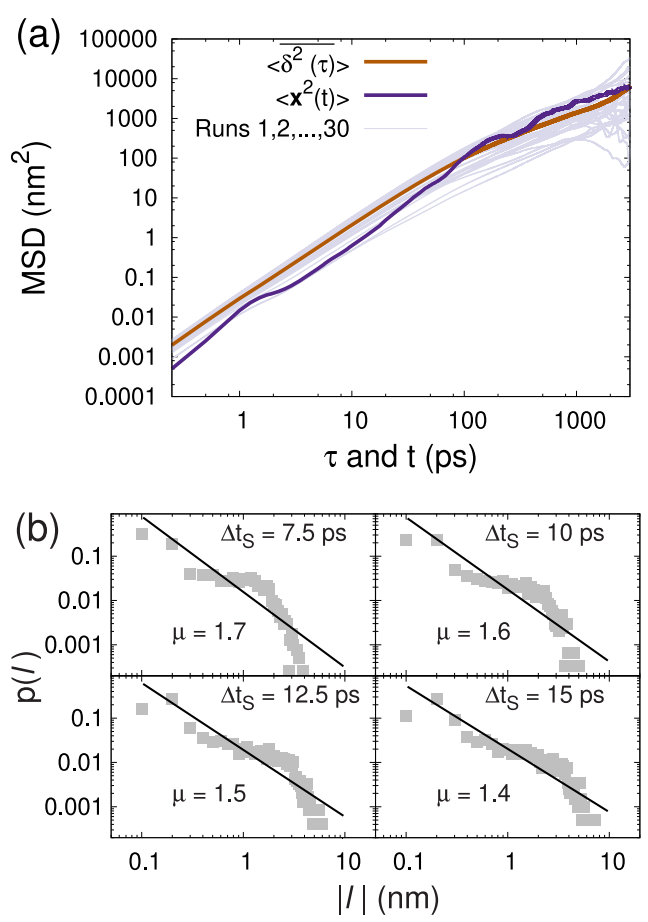

Figure 3. (a) Ensemble average $\left\langle\overline{\delta^{2}(\tau)}\right\rangle$ and $\left\langle x^{2}(t)\right\rangle$ for the $30 \mathrm{CMD}$ diffusion trajectories ( $3 \mathrm{~ns}$ long) and (b) jump length distribution for the $30 \mathrm{~ns}$ long CMD trajectory of $\mathrm{Ag}$ at $300 \mathrm{~K}$.

long-range adatom motion between diffusive domains (marked with circles in Figures 1 and S3a) as a random walk ${ }^{53}$ and hence extracting effective diffusivities from the Einstein equation. These diffusivities, however, cannot be directly correlated with static activation barriers as diffusive domains are not adsorption sites with an associated PEL. Moreover, the relevance of effective diffusivities should be seen in relation to the mean density of surface defects, ${ }^{54-56}$ because these defects may hinder the atomic motion and inhibit the transition to quasi-random walk behavior.

In summary, we study, via AIMD and CMD simulations, the diffusion of $\mathrm{Ag}, \mathrm{Au}, \mathrm{Cu}, \mathrm{Pd}, \mathrm{Pt}$, and $\mathrm{Ru}$ adatoms on monolayer graphene at $300 \mathrm{~K}$, a temperature that is relevant for thin-film synthesis experiments. We find that, while $\mathrm{Pt}$ and $\mathrm{Ru}$ exhibit typical features of a random walk, $\mathrm{Ag}, \mathrm{Au}, \mathrm{Cu}$, and $\mathrm{Pd}$ move continuously across the surface unhindered by adsorption sites, and follow a superdiffusive motion pattern known as Lévy walk. This motion is characterized by a wide distribution of jump lengths, often on the order of several nanometers. We also establish that the type of motion undergone by the adatoms is correlated with the PEL they experience on the SLG surface: diffusion resembles a random walk when the energy differences between adsorption sites are of the order of hundreds of millielectronvolts and turns into superdiffusive when these differences become of the order of $10 \mathrm{meV}$. The overall results of our work highlight that knowledge of static (i.e., $0 \mathrm{~K}$ ) surface migration barriers that are readily available $^{6-16}$ and govern the kinetics of random walks between adjacent adatom sites is not sufficient to describe the complex surface diffusion dynamics of adatoms on 2D-materials that exhibit flat PELs. Instead, modeling approaches that take into account the superdiffusive nature of adatom motion are required for guiding knowledge-based synthesis of metal-layers nanostructures with controlled morphology $y^{35,57-59}$ in nano- 
electronic, optoelectronic, and catalytic devices based on weakly interacting substrates. ${ }^{36-39}$

\section{COMPUTATIONAL METHODS}

We use AIMD simulations to study atomistic diffusion mechanisms with density functional theory (DFT) accuracy. All AIMD simulations are performed using the Vienna $a b$ initio simulation package (VASP). ${ }^{60}$ Core electrons are replaced by the projector augmented wave (PAW) pseudopotentials and the generalized gradient approximation of Perdew, Burke, and Ernzerhof (PBE). ${ }^{61}$ We use an energy cutoff of $400 \mathrm{eV}$ for the plane waves and a $3 \times 3 \times 1 \Gamma$-centered k-point mesh for the Brillouin zone integration. The monolayer graphene substrate is implemented as a hexagonal supercell containing 72 carbon atoms, between two vacuum layers of $10 \AA$ thickness, and periodic boundary conditions are established in all three directions. We model the van der Waals forces using the DFTD3 method with Becke-Johnson (BJ) damping, ${ }^{62}$ which accurately describes the properties of both graphite and diamond. $^{63}$ All simulations are spin-polarized. Initial test simulations showed that $\mathrm{Ru}$ and $\mathrm{Pt}$ exhibit diffusion patterns that are encountered in classical homoepitaxial growth systems, while $\mathrm{Ag}, \mathrm{Au}, \mathrm{Cu}$, and $\mathrm{Pd}$ diffuse considerably faster and execute jumps across multiple adsorption sites. Hence, we focus on $\mathrm{Ag}, \mathrm{Au}, \mathrm{Pd}$, and $\mathrm{Cu}$ adatoms, the diffusion of which is simulated for $0.2 \mathrm{~ns}$, while reference simulations are performed for $\mathrm{Pt}$ and $\mathrm{Ru}$ adatoms for times of 0.02 and $0.04 \mathrm{~ns}$, respectively.

We calculate the PEL at $0 \mathrm{~K}$ by computing the ground-state energies $E_{\text {ads }}$ of the monomers on the hollow, top, and bridge sites using standard DFT static structure relaxations, and the PEL at room temperature, by averaging $E_{\text {ads }}$ over the configurations of the AIMD simulations for which the monomer is found on each of the main adsorption sites.

AIMD runs are complemented by CMD simulations of $\mathrm{Ag}$ adatom diffusion on a graphene sheet of 680 atoms. These simulations are performed using LAMMPS. ${ }^{64}$ The $\mathrm{C}-\mathrm{C}$ and $\mathrm{Ag}-\mathrm{C}$ interactions are described using $\mathrm{AIREBO}^{65}$ and Lennard-Jones ${ }^{66}$ potentials, respectively. The AIREBO potential is well-established in the literature for simulating carbonbased materials ${ }^{67-70}$ and, together with Lennard-Jones, is used to study the diffusion of noble metals (including $\mathrm{Ag}$ ) clusters on graphite surfaces. ${ }^{71}$ More information about CMD validation can be found in the Supporting Information. We run a long simulation of $30 \mathrm{~ns}$ and 30 statistically independent simulations of $3 \mathrm{~ns}$, both with a time step of $0.25 \mathrm{fs}$. We use canonical sampling (Nosé-Hoover thermostat) of the configurational space for all $\mathrm{MD}$ simulations. AIMD and CMD outputs are visualized using the Ovito freeware. ${ }^{72}$

\section{ASSOCIATED CONTENT}

\section{SI Supporting Information}

The Supporting Information is available free of charge at https://pubs.acs.org/doi/10.1021/acs.jpclett.0c02375.

Comparison of $0.02 \mathrm{~ns}$ long AIMD trajectories (S1); calculation of scaling exponent $\alpha$ (S2); high-accuracy calculation of static ( $0 \mathrm{~K}$ ) adsorption energies (S3); entropy effects on diffusion dynamics (S4); potential energy landscape using classical interatomic potentials and CMD simulation analysis (S5) (PDF)

\section{AUTHOR INFORMATION}

\section{Corresponding Author}

Victor Gervilla - Nanoscale Engineering Division, Department of Physics, Chemistry and Biology, Linköping University, SE 58183 Linköping, Sweden; ○ orcid.org/0000-0003-27594147; Email: victor.gervilla@liu.se

\section{Authors}

Mohammad Zarshenas - Nanoscale Engineering Division, Department of Physics, Chemistry and Biology, Linköping University, SE 58183 Linköping, Sweden

Davide G. Sangiovanni - Theoretical Physics Division, Department of Physics, Chemistry and Biology, Linkoping University, SE 58183 Linköping, Sweden; @ orcid.org/00000002-1379-6656

Kostas Sarakinos - Nanoscale Engineering Division, Department of Physics, Chemistry and Biology, Linköping University, SE 58183 Linköping, Sweden; @ orcid.org/00000003-2864-9509

Complete contact information is available at:

https://pubs.acs.org/10.1021/acs.jpclett.0c02375

\section{Notes}

The authors declare no competing financial interest.

\section{ACKNOWLEDGMENTS}

V.G. acknowledges Mr. Davide Gambino (Linköping University) for useful discussions regarding spin polarization. V.G., M.Z., and K.S. acknowledge Linköping University ("LiU Career Contract, Dnr-LiU-2015-01510, 2015-2020"), the Swedish research council (contract VR-2015-04630) and the ÅForsk foundation (contract ÅF 19-137) for financial support. V.G., M.Z., D.S., and K.S. acknowledge the Olle Engkvist foundation (contract SOEB 190-312) for financial support. Simulations and data handling were enabled by resources provided by the Swedish National Infrastructure for Computing (SNIC) at the National Supercomputer Centre (NSC) partially funded by the Swedish Research Council through Grant Agreement No. VR-2015-04630.

\section{REFERENCES}

(1) Allain, A.; Kang, J.; Banerjee, K.; Kis, A. Electrical Contacts to Two-Dimensional Semiconductors. Nat. Mater. 2015, 14, 11951205.

(2) Schulman, D. S.; Arnold, A. J.; Das, S. Contact Engineering for 2D Materials and Devices. Chem. Soc. Rev. 2018, 47, 3037-3058.

(3) Petrov, I.; Barna, P. B.; Hultman, L.; Greene, J. E. Microstructural Evolution During Film Growth. J. Vac. Sci. Technol., A 2003, 21, S117-S128.

(4) Sarakinos, K. A Review on Morphological Evolution of Thin Metal Films on Weakly-Interacting Substrates. Thin Solid Films 2019, 688, 137312.

(5) Antczak, G.; Ehrlich, G. Surface Diffusion: Metals, Metal Atoms, and Clusters; Cambridge University Press: Cambridge, U.K., 2010.

(6) Tang, Y.; Zhang, H.; Shen, Z.; Zhao, M.; Li, Y.; Dai, X. The Electronic and Diffusion Properties of Metal Adatoms on Graphene Sheets: a First-Principles Study. RSC Adv. 2017, 7, 33208-33218.

(7) Amft, M.; Lebegue, S.; Eriksson, O.; Skorodumova, N. Adsorption of $\mathrm{Cu}, \mathrm{Ag}$, and $\mathrm{Au}$ Atoms on Graphene Including van der Waals Interactions. J. Phys.: Condens. Matter 2011, 23, 395001.

(8) Hupalo, M.; Liu, X.; Wang, C.-Z.; Lu, W.-C.; Yao, Y.-X.; Ho, K.M.; Tringides, M. C. Metal Nanostructure Formation on Graphene: Weak versus Strong Bonding. Adv. Mater. 2011, 23, 2082-2087. 
(9) Appy, D. V.; Lei, H.; Wang, C. Z.; Tringides, M. C.; Liu, D.-J.; Evans, J. W.; Thiel, P. A. Transition Metals on the (0001) Surface of Graphite: Fundamental Aspects of Adsorption, Diffusion, and Morphology. Prog. Surf. Sci. 2014, 89, 219-238.

(10) Manadé, M.; Viñes, F.; Illas, F. Transition Metal Adatoms on Graphene: a Systematic Density Functional Study. Carbon 2015, 95, 525-534.

(11) Han, Y.; Evans, J. Adsorption and Diffusion of Ru Adatoms on $\mathrm{Ru}(0001)$-supported Graphene: Large-Scale First-Principles Calculations. J. Chem. Phys. 2015, 143, 164706.

(12) Sun, X.; Wang, Z.; Fu, Y. Q. Adsorption and Diffusion of Sodium on Graphene with Grain Boundaries. Carbon 2017, 116, 415-421.

(13) Hasegawa, S.; Kunisada, Y.; Sakaguchi, N. Diffusion of a Single Platinum Atom on Light-Element-Doped Graphene. J. Phys. Chem. C 2017, 121, 17787-17795.

(14) Tang, Y.; Chen, W.; Li, C.; Li, W.; Dai, X.-Q. Geometric Stability, Electronic Structure, and Intercalation Mechanism of Co Adatom Anchors on Graphene Sheets. J. Phys.: Condens. Matter 2015, 27,255009

(15) Liu, X.; Wang, C. Z.; Hupalo, M.; Yao, Y. X.; Tringides, M. C.; Lu, W. C.; Ho, K. M. Adsorption and Growth Morphology of RareEarth Metals on Graphene Studied by Ab Initio Calculations and Scanning Tunneling Microscopy. Phys. Rev. B: Condens. Matter Mater. Phys. 2010, 82, 245408.

(16) Suarez-Martinez, I.; Felten, A.; Pireaux, J. J.; Bittencourt, C.; Ewels, C. P. Transition Metal Deposition on Graphene and Carbon Nanotubes. J. Nanosci. Nanotechnol. 2009, 9, 6171-6175.

(17) Eyring, H. The Activated Complex in Chemical Reactions. J. Chem. Phys. 1935, 3, 107-115.

(18) Vineyard, G. H. Frequency Factors and Isotope Effects in Solid State Rate Processes. J. Phys. Chem. Solids 1957, 3, 121-127.

(19) Michely, T.; Krug, J. Islands, Mounds and Atoms; SpringerVerlag: Berlin, 2004.

(20) Chen, L. Y.; Ying, S. C. Dynamics of Adatoms on Solid Surfaces. Phys. Rev. B: Condens. Matter Mater. Phys. 1994, 49, 1383813847.

(21) Ferrón, J.; Miranda, R.; de Miguel, J. J. Atomic Jumps During Surface Diffusion. Phys. Rev. B: Condens. Matter Mater. Phys. 2009, 79, 245407.

(22) Antczak, G.; Ehrlich, G. Long Jumps in Diffusion of Iridium on W(110). Phys. Rev. B: Condens. Matter Mater. Phys. 2005, 71, 115422.

(23) Senft, D. C.; Ehrlich, G. Long Jumps in Surface Diffusion: OneDimensional Migration of Isolated Adatoms. Phys. Rev. Lett. 1995, 74, 294-297.

(24) Sangiovanni, D.; Edström, D.; Hultman, L.; Petrov, I.; Greene, J.; Chirita, V. Ti Adatom Diffusion on $\operatorname{TiN}(001)$ : Ab Initio and Classical Molecular Dynamics Simulations. Surf. Sci. 2014, 627, 3441.

(25) Sangiovanni, D. Copper Adatom, Admolecule Transport, and Island Nucleation on TiN(001) via Ab Initio Molecular Dynamics. Appl. Surf. Sci. 2018, 450, 180-189.

(26) Kürpick, U.; Kara, A.; Rahman, T. S. Role of Lattice Vibrations in Adatom Diffusion Ulrike. Phys. Rev. Lett. 1997, 78, 1086-1089.

(27) Boisvert, G.; Mousseau, N.; Lewis, L. J. Comment on "Role of Lattice Vibrations in Adatom Diffusion. Phys. Rev. Lett. 1998, 80, 203-203.

(28) Kürpick, U.; Kara, A.; Rahman, T. S.; et al. Kürpick et al. Reply. Phys. Rev. Lett. 1998, 80, 204-204.

(29) Mei, A. B.; Hellman, O.; Wireklint, N.; Schlepütz, C. M.; Sangiovanni, D. G.; Alling, B.; Rockett, A.; Hultman, L.; Petrov, I.; Greene, J. E. Dynamic and Structural Stability of Cubic Vanadium Nitride. Phys. Rev. B: Condens. Matter Mater. Phys. 2015, 91, No. 054101.

(30) Sangiovanni, D. G.; Mei, A. B.; Edström, D.; Hultman, L.; Chirita, V.; Petrov, I.; Greene, J. E. Effects of Surface Vibrations on Interlayer Mass Transport: Ab Initio Molecular Dynamics Investigation of Ti Adatom Descent Pathways and Rates from TiN/
TiN(001) Islands. Phys. Rev. B: Condens. Matter Mater. Phys. 2018, 97, No. 035406.

(31) Smirnova, D.; Starikov, S.; Leines, G. D.; Liang, Y.; Wang, N.; Popov, M. N.; Abrikosov, I. A.; Sangiovanni, D. G.; Drautz, R.; Mrovec, M. Atomistic Description of Self-Diffusion in Molybdenum: A Comparative Theoretical Study of Non-Arrhenius Behavior. Phys. Rev. Mater. 2020, 4, No. 013605.

(32) Iacopi, F.; Boeckl, J. J.; Jagadish, C. 2D Materials; Elsevier, 2016; Chapter 1, pp 1-33.

(33) Noori, K.; Cheng, N.; Xuan, F.; Quek, S. Dielectric Screening by 2D Substrates. 2D Mater. 2019, 6, No. 035036.

(34) Novoselov, K. S.; Geim, A. K.; Morozov, S. V.; Jiang, D.; Zhang, Y.; Dubonos, S. V.; Grigorieva, I. V.; Firsov, A. A. Electric Field Effect in Atomically Thin Carbon Films. Science 2004, 306, 666-669.

(35) Liu, X.; Han, Y.; Evans, J. W.; Engstfeld, A. K.; Behm, R. J.; Tringides, M. C.; Hupalo, M.; Lin, H.-Q.; Huang, L.; Ho, K.-M.; et al. Growth Morphology and Properties of Metals on Graphene. Prog. Surf. Sci. 2015, 90, 397-443.

(36) Lee, J.-H.; Choi, H. K.; Yang, L.; Chueng, S.-T. D.; Choi, J.-W.; Lee, K.-B. Non-Destructive Real-Time Monitoring of Enhanced Stem Cell Differentiation using a Graphene-Au Hybrid Nanoelectrode Array. Adv. Mater. 2018, 30, 1802762.

(37) Yuwen, L.; Xu, F.; Xue, B.; Luo, Z.; Zhang, Q.; Bao, B.; Su, S.; Weng, L.; Huang, W.; Wang, L. General Synthesis of Noble Metal $(\mathrm{Au}, \mathrm{Ag}, \mathrm{Pd}, \mathrm{Pt})$ Nanocrystal Modified MoS2 Nanosheets and the Enhanced Catalytic Activity of PdMoS2 for Methanol Oxidation. Nanoscale 2014, 6, 5762-5769.

(38) Tan, C.; Huang, X.; Zhang, H. Synthesis and Applications of Graphene-Based Noble Metal Nanostructures. Mater. Today 2013, 16, $29-36$.

(39) Chae, S. S.; Jang, S.; Lee, W.; Jung, D. W.; Lee, K. H.; Kim, J. D.; Jeong, D.; Chang, H.; Hwang, J. Y.; Lee, J.-O. Ultrathin Metal Crystals: Growth on Supported Graphene Surfaces and Applications. Small 2018, 14, 1801529.

(40) Saxton, M. J. Single-Particle Tracking: The Distribution of Diffusion Coefficients. Biophys. J. 1997, 72, 1744-1753.

(41) Shlesinger, M.; Zaslavsky, G.; Klafter, J. Strange Kinetics. Nature 1993, 363, 31-37.

(42) Metzler, R.; Jeon, J.-H.; Cherstvy, A. G.; Barkai, E. Anomalous Diffusion Models and their Properties: Non-Stationarity, NonErgodicity, and Ageing at the Centenary of Single Particle Tracking. Phys. Chem. Chem. Phys. 2014, 16, 24128-24164.

(43) Kepten, E.; Weron, A.; Sikora, G.; Burnecki, K.; Garini, Y. Guidelines for the Fitting of Anomalous Diffusion Mean Square Displacement Graphs from Single Particle Tracking Experiments. PLoS One 2015, 10, e0117722.

(44) Lii-Rosales, A.; Han, Y.; Jing, D.; Tringides, M. C.; Thiel, P. A. Search for Encapsulation of Platinum, Silver, and Gold at the Surface of Graphite. Phys. Rev. Research 2020, 2, No. 033175.

(45) Lii-Rosales, A.; Han, Y.; Yu, K. M.; Jing, D.; Anderson, N.; Vaknin, D.; Tringides, M. C.; Evans, J. W.; Altman, M. S.; Thiel, P. A. Reverse-Engineering of Graphene on Metal Surfaces: a Case Study of Embedded Ruthenium. Nanotechnology 2018, 29, 505601.

(46) Zakharchenko, K.; Katsnelson, M.; Fasolino, A. Finite Temperature Lattice Properties of Graphene beyond the Quasiharmonic Approximation. Phys. Rev. Lett. 2009, 102, No. 046808.

(47) Frenkel, D.; Smit, B. Understanding Molecular Simulation: From Algorithms to Applications; Academic Press: San Diego, CA, 1996.

(48) Zaburdaev, V.; Denisov, S.; Klafter, J. Lévy Walks. Rev. Mod. Phys. 2015, 87, 483-530.

(49) Barthelemy, P.; Bertolotti, J.; Wiersma, D. A Lévy Flight for Light. Nature 2008, 453, 495-498.

(50) Humphries, N.; et al. Environmental Context Explains Levy and Brownian Movement Patterns of Marine Predators. Nature 2010, $465,1066-1069$.

(51) Brockmann, D.; Hufnagel, L.; Geisel, T. The Scaling Laws of Human Travel. Nature 2006, 439, 462-465. 
(52) Maruyama, Y.; Murakami, J. Truncated Lévy Walk of a Nanocluster Bound Weakly to an Atomically Flat Surface: Crossover from Superdiffusion to Normal Diffusion. Phys. Rev. B: Condens. Matter Mater. Phys. 2003, 67, No. 085406.

(53) Sancho, J. M.; Lacasta, A. M.; Lindenberg, K.; Sokolov, I. M.; Romero, A. H. Diffusion on a Solid Surface: Anomalous is Normal. Phys. Rev. Lett. 2004, 92, 250601.

(54) Cançado, L. G.; Jorio, A.; Ferreira, E. H. M.; Stavale, F.; Achete, C. A.; Capaz, R. B.; Moutinho, M. V. O.; Lombardo, A.; Kulmala, T. S.; Ferrari, A. C. Quantifying Defects in Graphene via Raman Spectroscopy at Different Excitation Energies. Nano Lett. 2011, 11, 3190-3196.

(55) Lucchese, M.; Stavale, F.; Ferreira, E. M.; Vilani, C.; Moutinho, M.; Capaz, R. B.; Achete, C.; Jorio, A. Quantifying Ion-Induced Defects and Raman Relaxation Length in Graphene. Carbon 2010, 48, 1592-1597.

(56) Dresselhaus, M. S.; Jorio, A.; Souza Filho, A. G.; Saito, R. Defect Characterization in Graphene and Carbon Nanotubes using Raman Spectroscopy. Philos. Trans. R. Soc., A 2010, 368, 5355-5377.

(57) Lü, B.; Almyras, G. A.; Gervilla, V.; Greene, J. E.; Sarakinos, K. Formation and Morphological Evolution of Self-Similar 3D Nanostructures on Weakly Interacting Substrates. Phys. Rev. Mater. 2018, 2, No. 063401.

(58) Gervilla, V.; Almyras, G. A.; Thunström, F.; Greene, J. E.; Sarakinos, K. Dynamics of 3D-Island Growth on Weakly-Interacting Substrates. Appl. Surf. Sci. 2019, 488, 383-390.

(59) Gervilla, V.; Almyras, G. A.; Lü, B.; Sarakinos, K. Coalescence Dynamics of 3D Islands on Weakly-Interacting Substrates. Sci. Rep. 2020, 10, 2031.

(60) Kresse, J.; Furthmüller, J. Efficiency of Ab Initio Total Energy Calculations for Metals and Semiconductors Using a Plane-Wave Basis Set. Comput. Mater. Sci. 1996, 6, 15-50.

(61) Perdew, J.; Burke, K.; Ernzerhof, M. Generalized Gradient Approximation Made Simple. Phys. Rev. Lett. 1996, 77, 3865-3868.

(62) Grimme, S.; Ehrlich, S.; Goerigk, L. Effect of the Damping Function in Dispersion Corrected Density Functional Theory. J. Comput. Chem. 2011, 32, 1456-1465.

(63) Mosyagin, I.; Gambino, D.; Sangiovanni, D.; Abrikosov, I.; Caffrey, N. Effect of Dispersion Corrections on Ab Initio Predictions of Graphite and Diamond Properties Under Pressure. Phys. Rev. B: Condens. Matter Mater. Phys. 2018, 98, 174103.

(64) Plimpton, S. Fast Parallel Algorithms for Short-Range Molecular Dynamics. J. Comput. Phys. 1995, 117, 1-19.

(65) Stuart, S. J.; Tutein, A. B.; Harrison, J. A. A Reactive Potential for Hydrocarbons with Intermolecular Interactions. J. Chem. Phys. 2000, 112, 6472-6486.

(66) Neek-Amal, M.; Asgari, R.; Tabar, M. R. R. The Formation of Atomic Nanoclusters on Graphene Sheets. Nanotechnology 2009, 20, 135602.

(67) Varshney, V.; Patnaik, S. S.; Roy, A. K.; Froudakis, G.; Farmer, B. L. Modeling of Thermal Transport in Pillared-Graphene Architectures. ACS Nano 2010, 4, 1153-1161.

(68) Safina, L.; Baimova, J.; Mulyukov, R. Nickel Nanoparticles inside Carbon Nanostructures: Atomistic Simulation. Mech. Adv. Mater. Mod. Process 2019, 5, 2.

(69) Koukaras, E.; Kalosakas, G.; Galiotis, C.; Papagelis, K. Phonon Properties of Graphene Derived from Molecular Dynamics Simulations. Sci. Rep. 2015, 5, 12923.

(70) Liu, B.; Reddy, C. D.; Jiang, J.; Baimova, J. A.; Dmitriev, S. V.; Nazarov, A. A.; Zhou, K. Morphology and In-Plane Thermal Conductivity of Hybrid Graphene Sheets. Appl. Phys. Lett. 2012, 101, 211909.

(71) Yousefi, P.; Abbaspour, M.; Sokhanvaran, V. A Comparative Study of Graphite and CNT Supported Au-Ag, Au-Pd, Au-Pt and AuRh Nanoalloys using MD Simulation. J. Mol. Liq. 2019, 280, 87-96.

(72) Stukowski, A. Visualization and Analysis of Atomistic Simulation Data with OVITO the Open Visualization Tool. Modell. Simul. Mater. Sci. Eng. 2010, 18, No. 015012. 